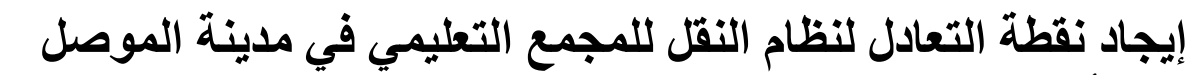 \\ ساره عدنان سعيد

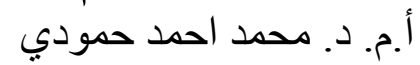 \\ جامعة الموصل"/كلية الهندسة/ قمم الهندسة المدنية

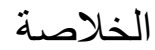

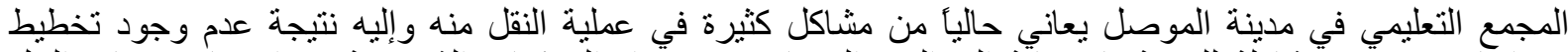

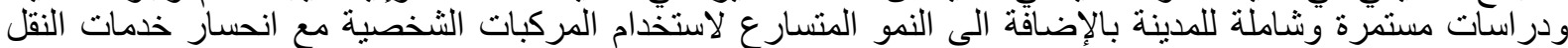

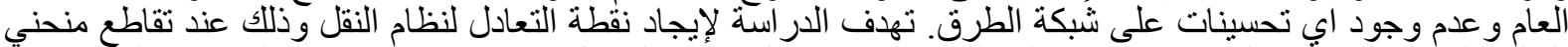

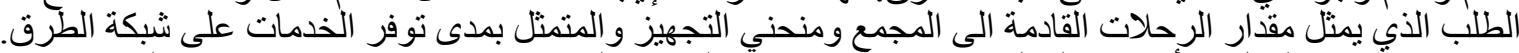

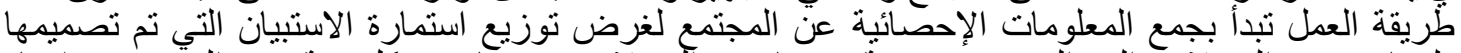

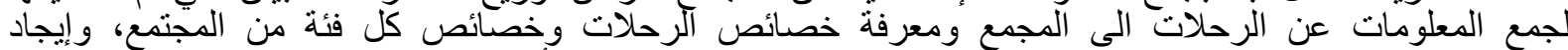

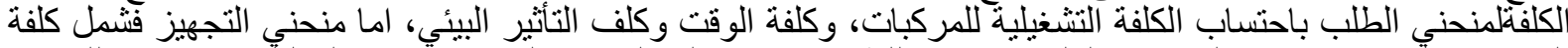

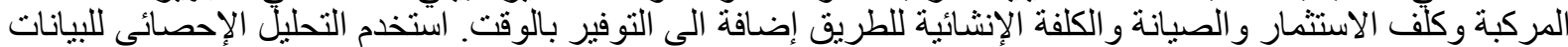

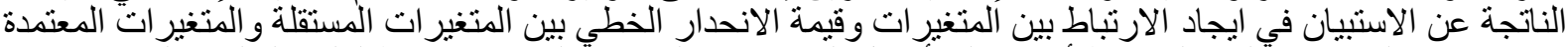

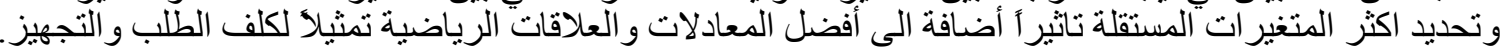

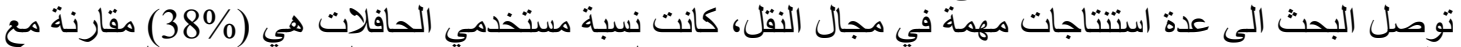

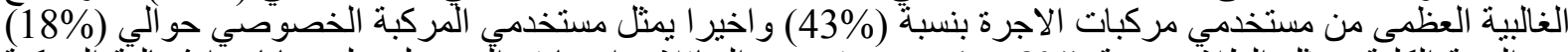

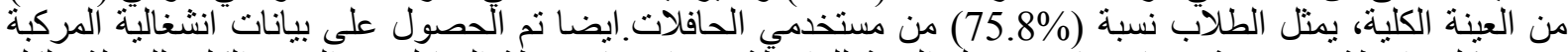

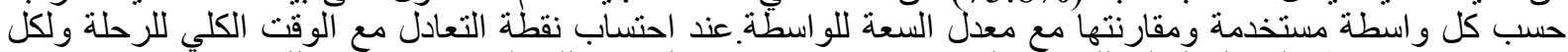

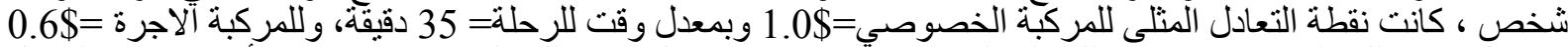
وبمعدل وقت للرحلة= 60 دقيقة، وللحافلة الصغيرة =

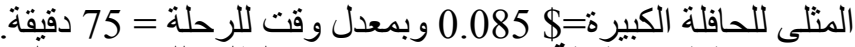

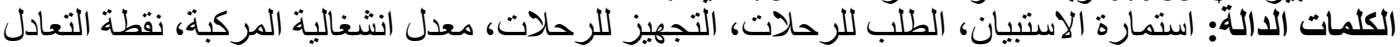

\section{Determination of Equilibrium Point for Transportation System of Educational Campus in Mosul City}

Sarah Adnan Saied

Dr. Mohammad Ahmed Humoody

University of Mosul/College of Engineering/Civil Engineering

\section{Abstract}

The Educational Campus in Mosul city is suffering from many problems with its transportation system ,due to lack of planning process and continuing studies either comprehensive one, in addition to the rapid growth of the use of personal vehicles with the decline in public transport services and the lack of any improvements of the road network too. The study aims to find out an equilibrium point of the transportation system at the intersection of the demand curve with supply curve, which represents the cost of services to be subsidized and availability of facilities on the network.

Methodology started with collecting data, which includes statistical information for the community for the purpose of sample size and sampling process. The distributed questionnaire forms that was designed to gather information on trips and the knowledge of the properties of trip characteristics was done. Then cost was found about the demand curve calculated operating costs for vehicles, in addition to the cost time and cost environmental impact. Either curved processing evolved to include the cost of the vehicle and the cost of investment and maintenance, and cost of construction of the road in addition to the time savings . Statisticalanalysis was employed to find the correlation between variables. Linear regression between the independent variables and the dependent variables were identified as the most influential independent variables in addition to the best equations and mathematical relationships representative of the cost and demand processing. Research has come to several important conclusions to improve transport with the modes used . The proportion of public transit users are $(38 \%)$ compared with the majority of users of taxis by $(43 \%)$, and finally representing users of the passenger car user about (18\%) of the total sample , the representing students ratio $(75.8 \%)$ of the users of the bus, also obtained data vehicle occupancy according to each mode. The final results show that the equilibrium point for each person is 1.0 \$ for PC with average travel time $=35 \mathrm{~min}$, and $0.6 \$$ for taxi with travel time $=60 \mathrm{~min}$, and $0.32 \$$ for small bus with travel time $=105 \mathrm{~min}$, finally for large bus $=0.085 \$$ with travel time $=75 \mathrm{~min}$. key word: Travel Demand, Interview Survey Form, Average Vehicle Occupancy(AVO), Travel Supply, Equilibrium point. 
ان نظام النقل الحالي في مدينة الموصل يعاني من مشاكل كثيرة نتيجة عدم وجود عملية تخطبط مستمرة وشاملة للمدينة

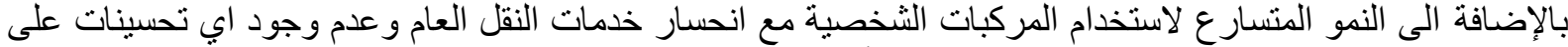

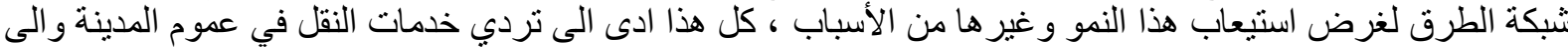

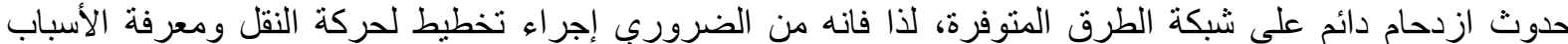

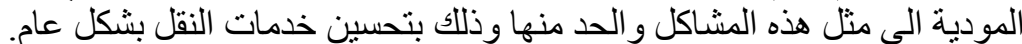

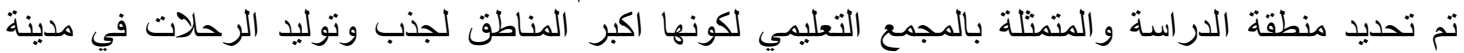

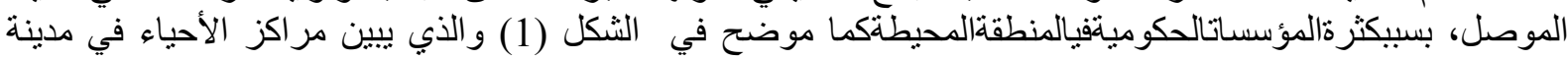

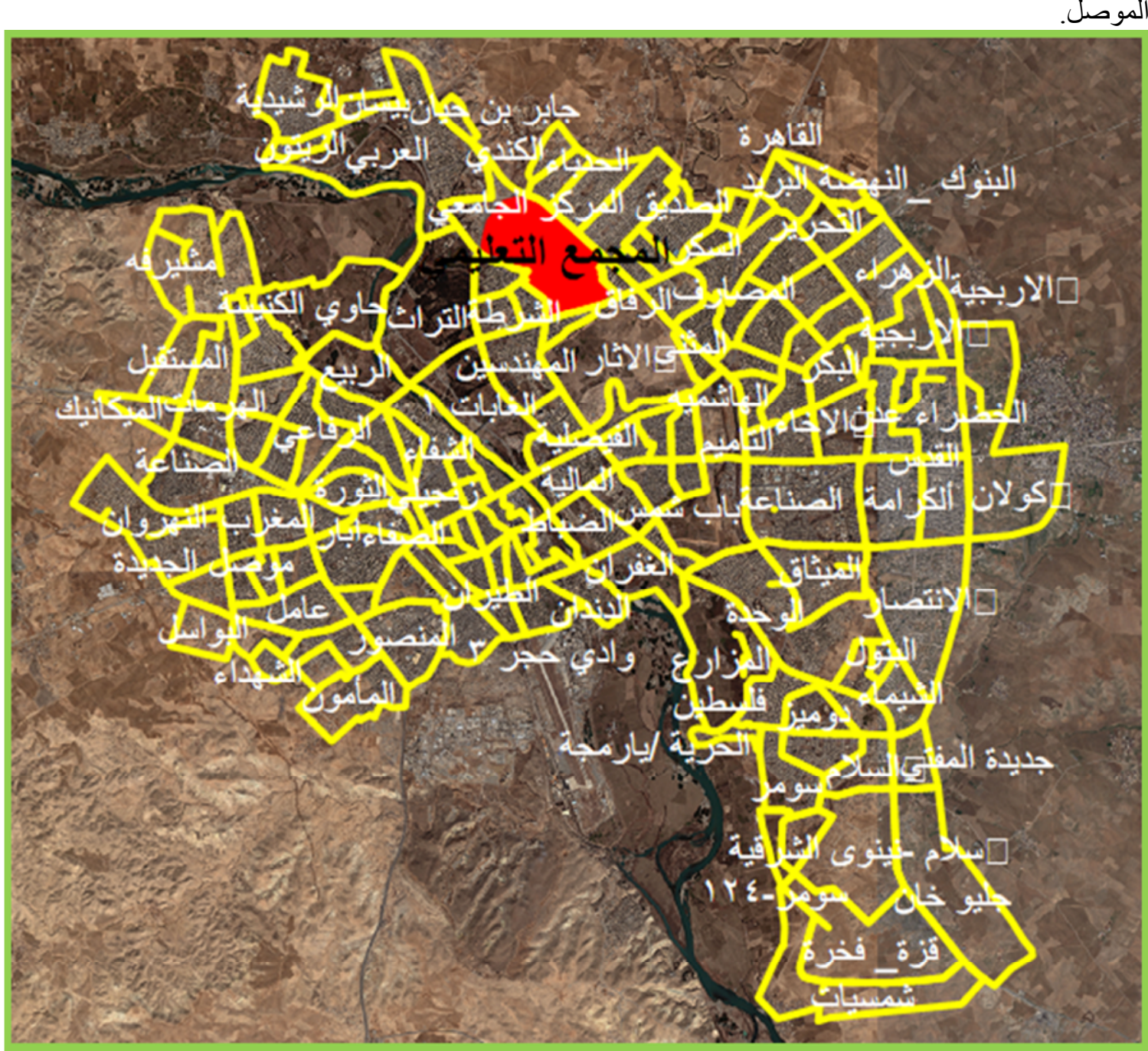

شكل(1) : منطقة الدر اسة ضمن أحياء مدينة الموصل حسب بيانات الدر اسة ،2013.

تكمن المشكلة في كون قطاع النقل العام لخدمات الحافلات المتوفرة بين المجمع التعليمي و المدينة محدود العدد المداد

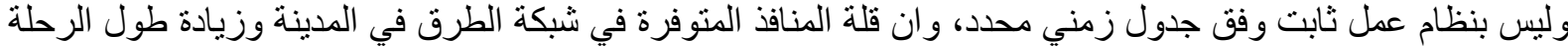

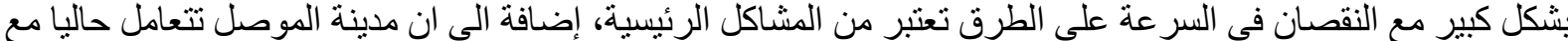

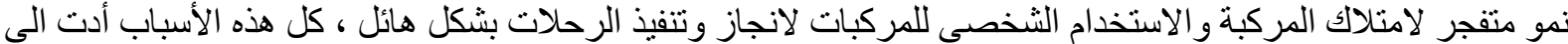

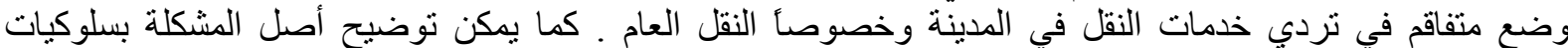
مستخدمي الطريق حيث عادة ما يكونون ذو حساسية للكلف الخفية للمركبة ، مثل سعر الثراء الثياء و التأمين والصيانة ،

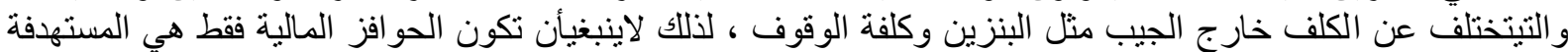
عند توفير وسائط بديلة للنقل الأكثر اغر اءآ للركاب ولكن ايضاً الحد من استخدام القيادة منفردا. 


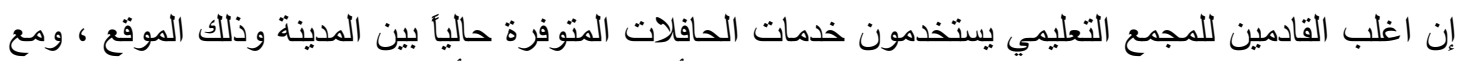

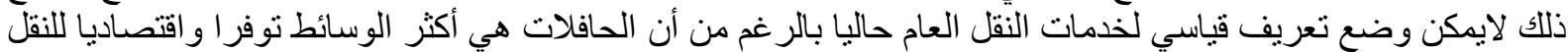

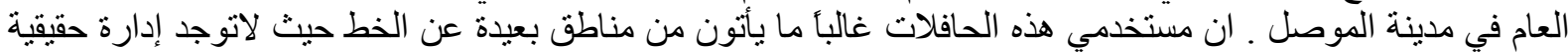
ل لخدمات الحالية للحافلات العوصل ان

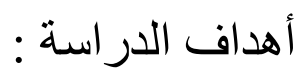

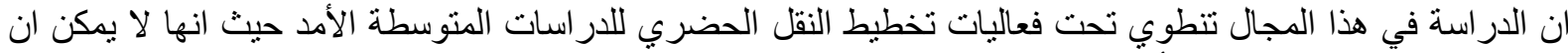

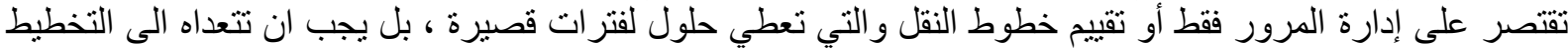

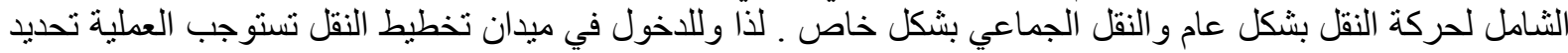

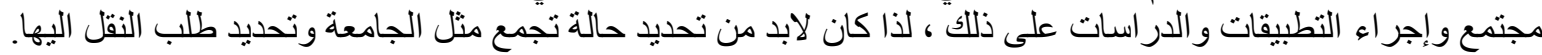

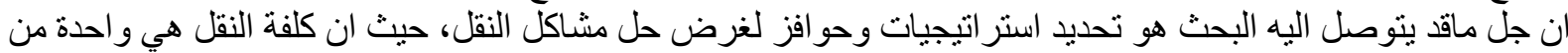

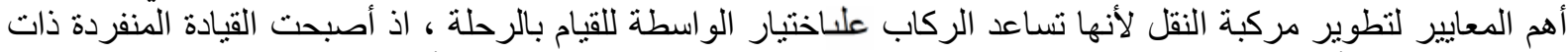

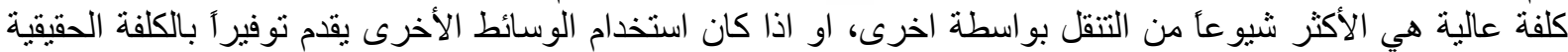

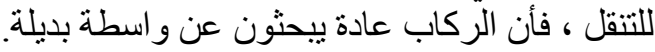

يهدف البحث للوصول الى منحنيات الطلب و التجهيز(Demand and Supply)التي تعتبر الأساس في اية عملية

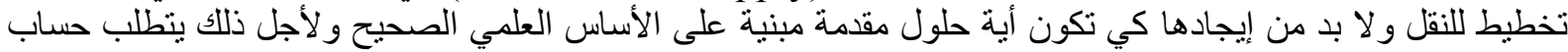

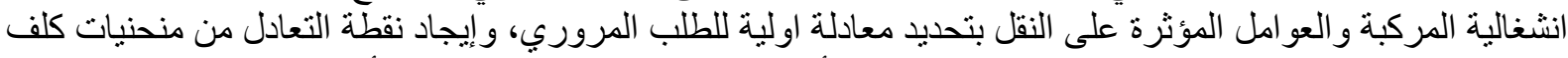

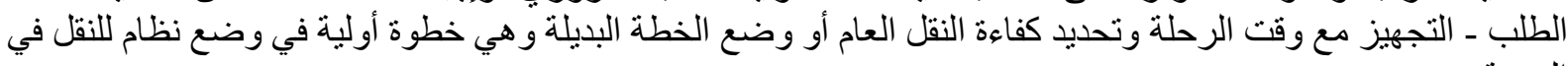
المدينة

$$
\text { نظرة في الادبيات: }
$$

هنالك عدة در اسات اجريت بخصوص النقل في العر اق وكانت لها أهداف مختلفة اعتمادا على المشكلة التي تو اجه

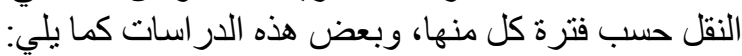

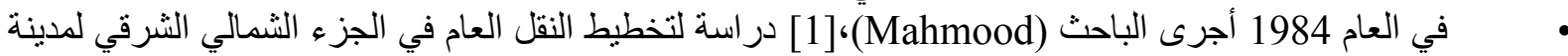

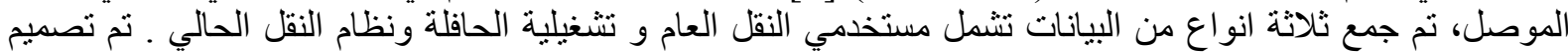

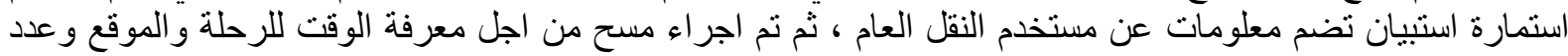
الركاب في مسار الدراسة ، بعد ذلك اجريت مقابلات مع الوكالات المسؤولة فئلة عن النقل العاء العام.

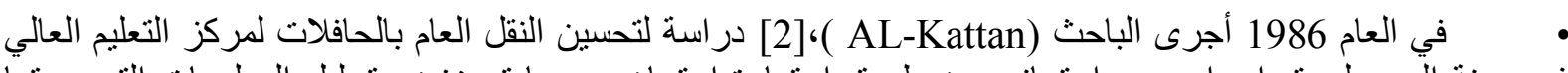

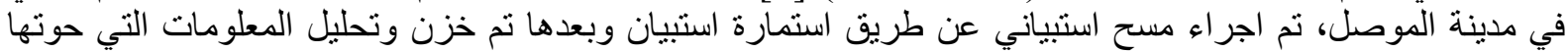

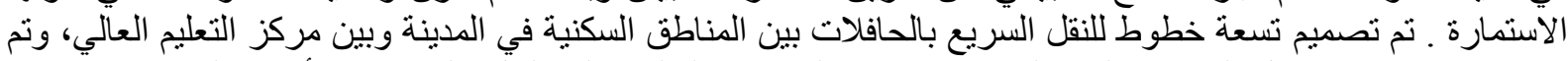

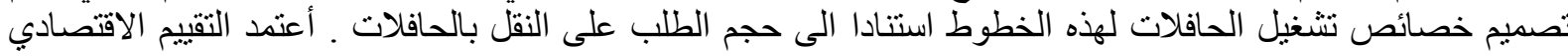

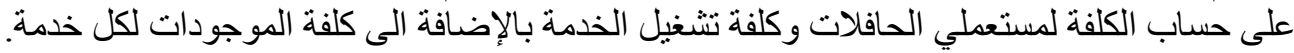

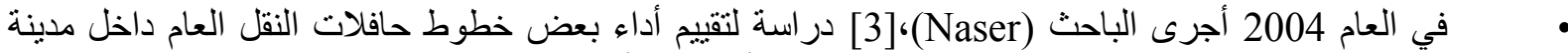

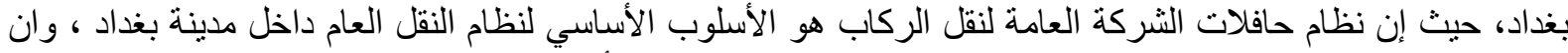

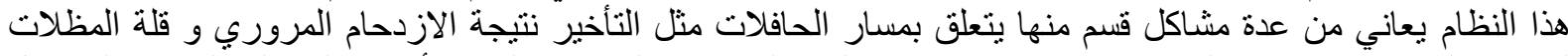

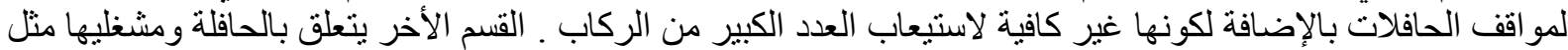

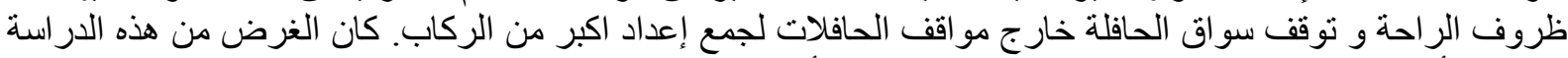

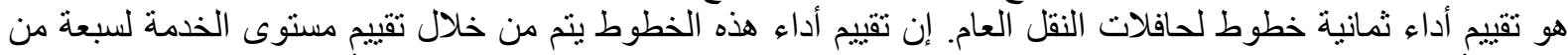

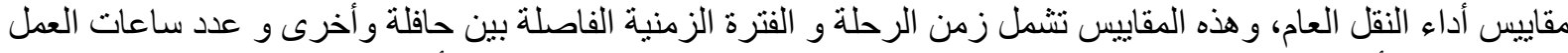

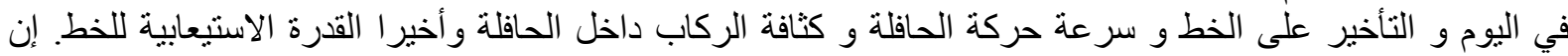

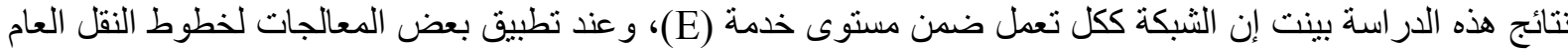

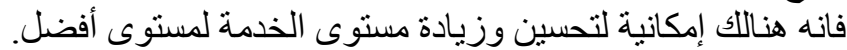




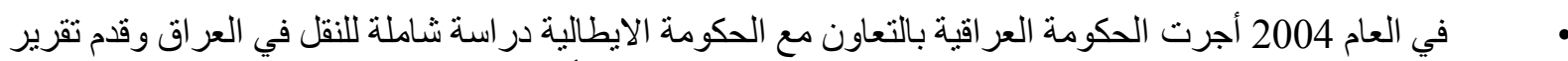

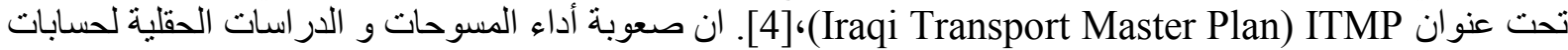

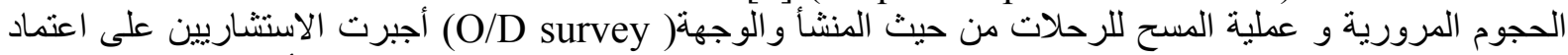

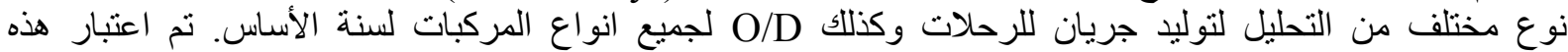

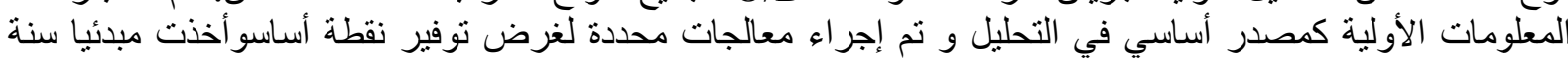

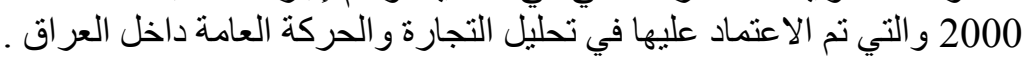

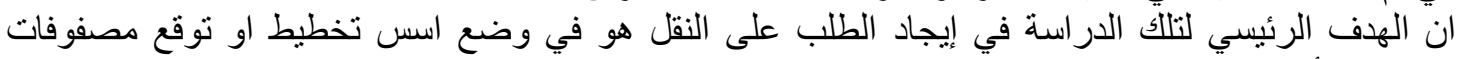

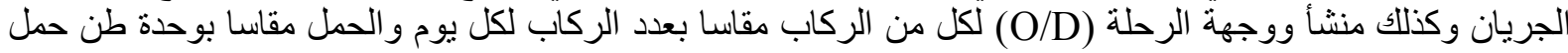

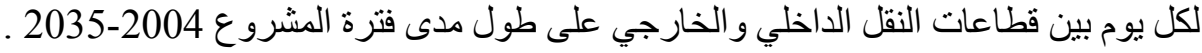

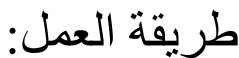

\section{لغرض الوصول الى أهداف الدراسة تم وضع إستر اتيجية لطريقة العمل [5]و التي تشمل مايلي:}

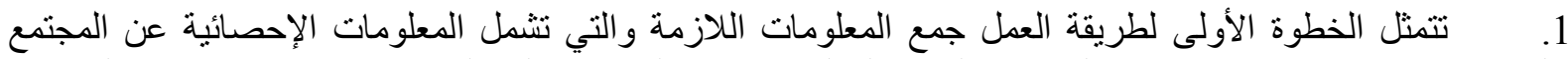

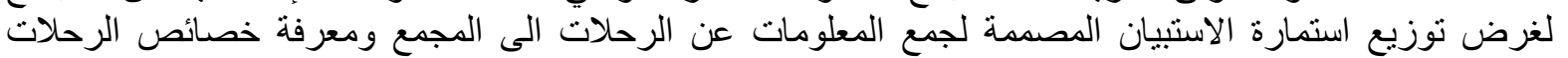

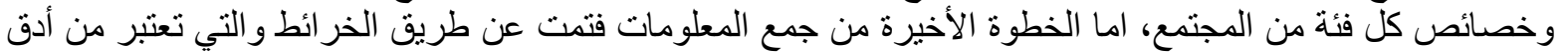

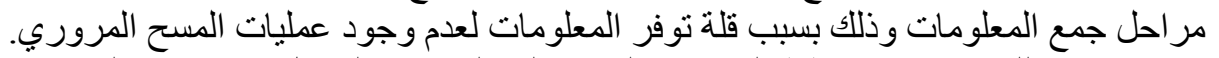

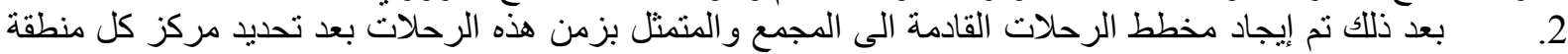

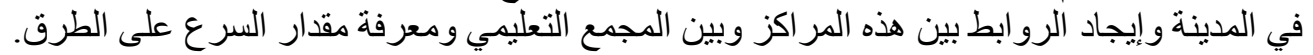
3.

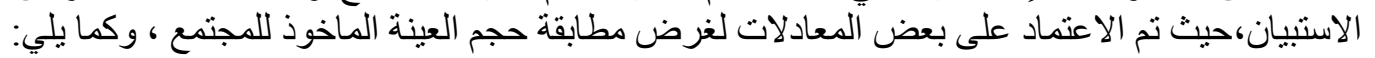

$$
\mathrm{n}=\frac{\mathrm{P}(1-\mathrm{P}) * \mathrm{~N}^{3}}{\left(\frac{\mathrm{E}}{1.96}\right)^{2} *(\mathrm{~N}-1)+\mathrm{P}(1-\mathrm{P}) * \mathrm{~N}^{2}}
$$

=n N=N E P = النسبة السكانية المتوقعة في المجتمع مع خاصية الفائدة= 0.5

$\mathrm{n}=\frac{\left(\frac{\mathrm{P}(1-\mathrm{P})}{\mathrm{A}^{2}+\frac{\mathrm{P}(1-\mathrm{P})}{\mathrm{N}}}\right)}{\mathrm{R}}$

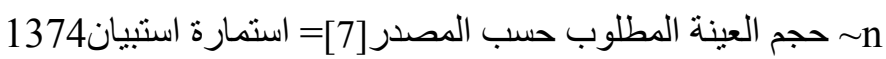

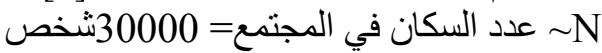

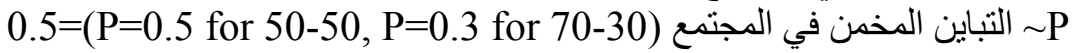
3\% = الدقة المطلوبة (10\% A

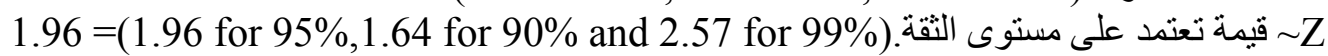

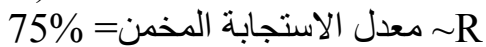

حيث كان حجم العينة الماخوذ = 3000 ويمثل حوالي (10\%) من المجتمع وهو يزيد عن اقصى قيمة للحجم المطلوب بمقار (40\%)، وذللك لتلافي حالات عدم الاسترجاع أو الاستجابة وكذللك لاعطاء مجال حول رهض رفض البيانات الثاذة.

4. بعد إجر اء عملية الاستبيان حسب حجم العينة نم حصر البيانات لمنطقة الدراسة وذلك بمعرفة نسب الاستجابة

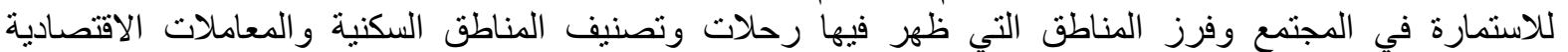

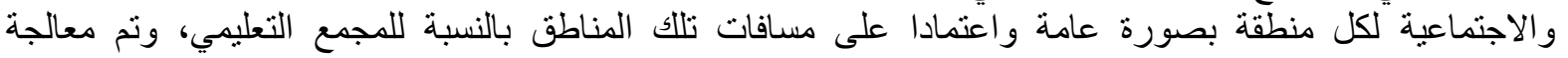
الاستمار ات التي اظهرت معلومات منحازة ، وذلك بموجب الاستمارة المعدة من قبل الباحث كما في شكل (2). 


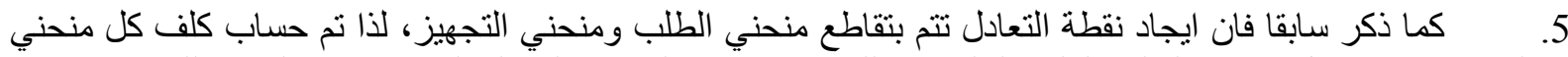
على حدة حيث شمل منحني الطلب الكلف التالية ، وذللك وفق معايير الدر اسة الثاملة التي تمت في العراق للفترة (20042035) وكما يلي: كلفة الطلب = (الكلفة التشغيلية) + كلفة الوقت + كلفة التأثير البيئي حيث ان (الكلفة التشغيلية) = كلف استهلاك الوقود + كلف استهلاك الإطار + كلف استهلاك زيت المحرك + كلف طاقم العمل و الصيانة + الكلفة الإضافية (النية وكما هو موضح في الجدول ( 1 ) لجميع انواعة المركبات التي استخدمت للوصول الى منطقة الدراسة. جدول (1 ) : تفاصيل كلف الطلب حسب نوع المركبة المستخدمة لوحدة رحلة واحدة

\begin{tabular}{|c|c|c|c|c|c|c|}
\hline كلف التأثير & و الفة طاقم & للنشبة الكلفة الإضافية & صرفيات & صرفيات & $\begin{array}{c}\text { صرفيات } \\
\text { الوقود } \\
\text { \$/km }\end{array}$ & نوع المركبة \\
\hline 0.00341 & 3.24 & 1.07 & 0.0049 & 0.004 & 0.0324 & مركبة خصوصي \\
\hline 0.00378 & 11.7 & 1.08 & 0.0049 & 0.004 & 0.0358 & اشتر الك أو أجرة \\
\hline 0.00618 & 9 & 1.08 & 0.00794 & 0.01 & 0.2467 & حافلة صغيرة \\
\hline 0.00264 & 9.9 & 1.1 & 0.00794 & 0.029 & 0.1028 & حافلة كبيرة \\
\hline
\end{tabular}

اما بالنسبة لكلف منحني التجهيز يشمل الكلف التالية،وكما موضح في الجدول (2) : كلفة التجهيز = كلفة المركبة + كلفة التوفير بالوقت + كلف الاستثمار والصيانة + الكلفة الإنثائية لمقطع الطريق جدول (2 ) : تفاصيل كلف التجهيز حسب نوع المركبة المستخدمة لوحدة رحلة واحدة

\begin{tabular}{|c|c|c|c|c|}
\hline $\begin{array}{c}\text { الكلفة الإنشائية } \\
\$ V k M T\end{array}$ & كلفة الاستثمار و الصيانة & نوع الطريق & سعر المركبة & نوع المركبة \\
\hline \multirow[t]{2}{*}{0.053} & \multirow{2}{*}{0.0714} & \multirow{2}{*}{$\mathrm{A} 2$} & 0.018 & مركبة خصوصي \\
\hline & & & 0.013 & اشتر الك او أجرة \\
\hline \multirow[t]{2}{*}{0.125} & \multirow{2}{*}{0.0589} & \multirow{2}{*}{ A6 } & 0.017 & حافلة صغيرة \\
\hline & & & 0.023 & حافلة كبيرة \\
\hline
\end{tabular}

كما انه من الجدير بالذكر ان كلفة الوقت لعام 2013 كانت 1.184 ـ / hr عند حساب الطلب للرحلة ، وكان قيمة

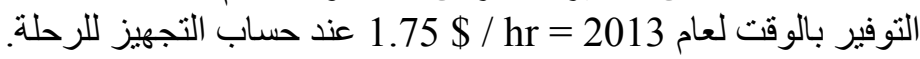

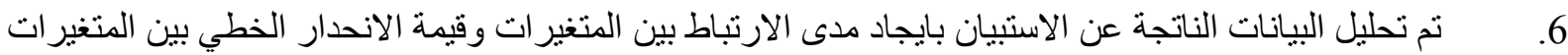

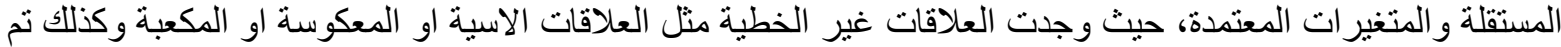

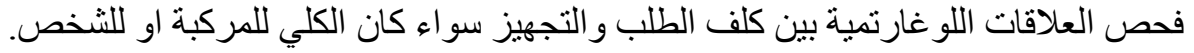
7.

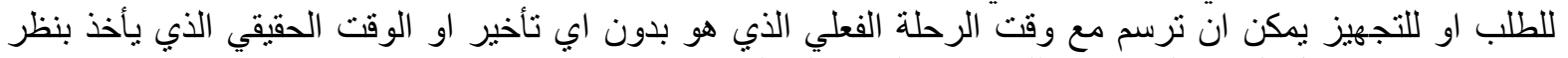

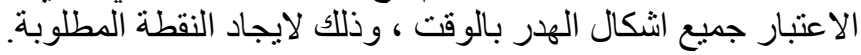


كلية الهنبسة

بزاسة انتقل العقام في جامعة أموصل

جامعة الموصل

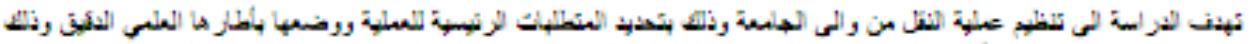

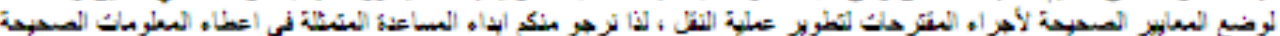

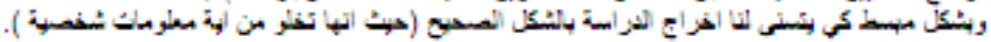
مي فابو المنكر والثقير

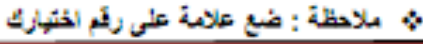

\begin{tabular}{|c|c|c|c|c|c|c|c|c|}
\hline & & & & كلم الاستعلزة: & & & قاحص & (" ") ) مغومدت تخص \\
\hline+ & ، خزع الجمعة & , & لغل الجأمية & (T1) المركي : & 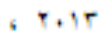 & 1 & 1 & (ז') تزري الاستبلن \\
\hline
\end{tabular}

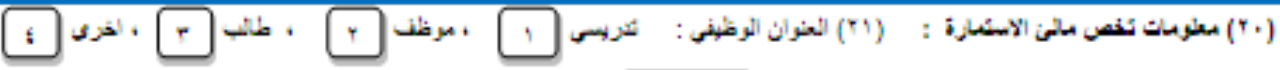

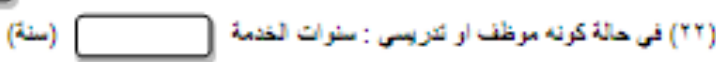

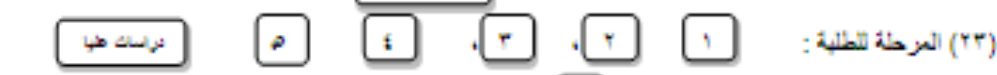

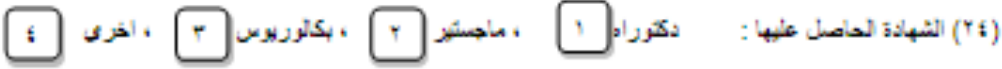

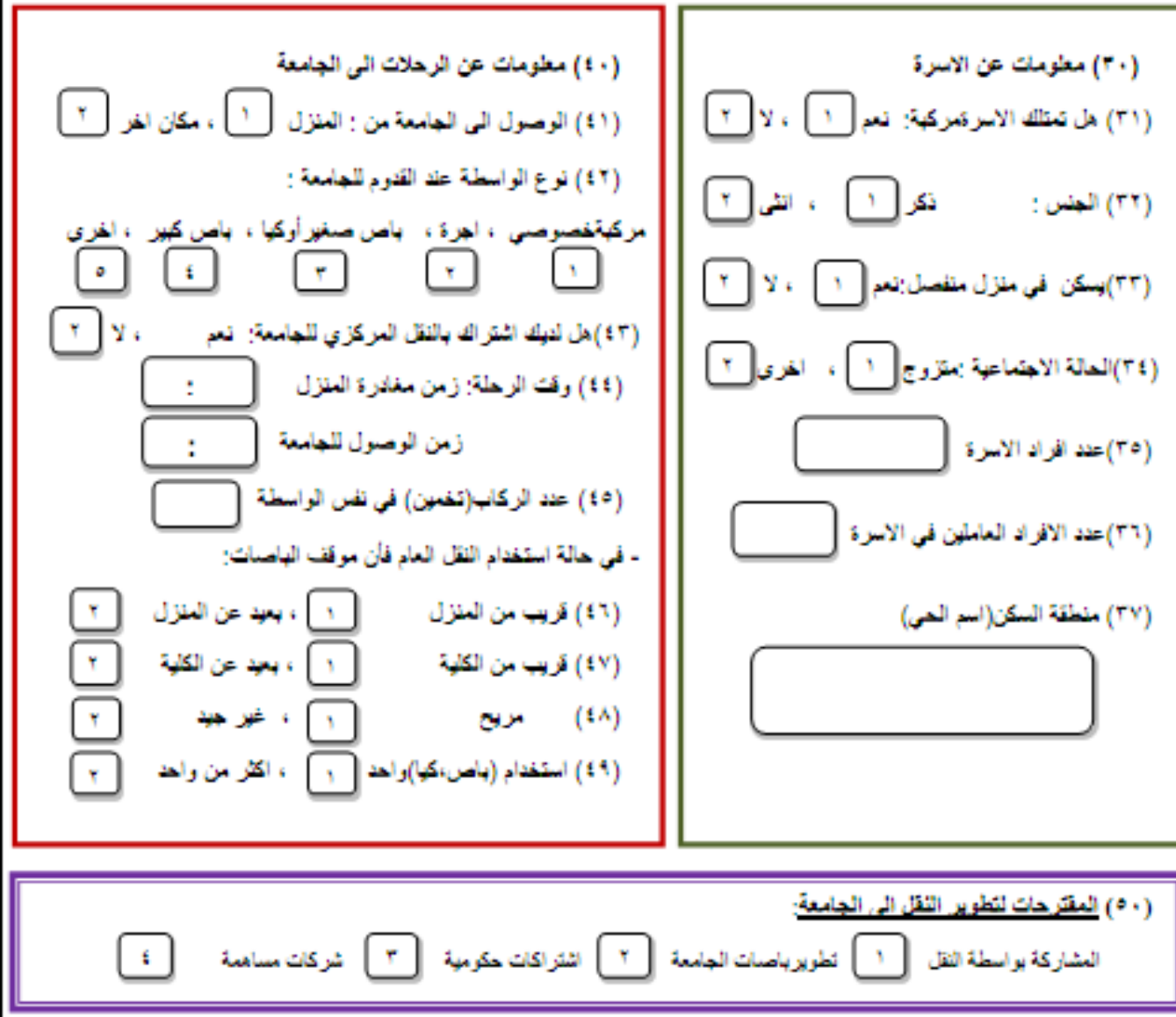

شكل (2) : استمارة الاستبيان لدر اسة النقل المعدة من قبل الباحث، 2013

ان من اهم خطوات التحليل للرحلات في عملية تخطيط النقل هي عملية فصل استخدام الواسطة للنقل، حيث ان موديل

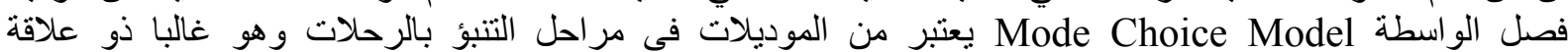

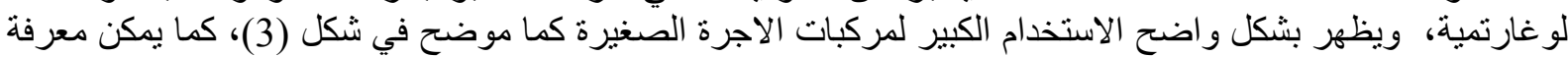

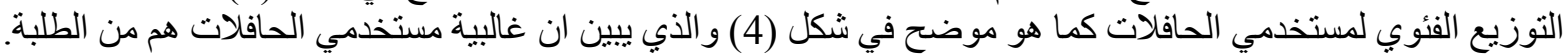




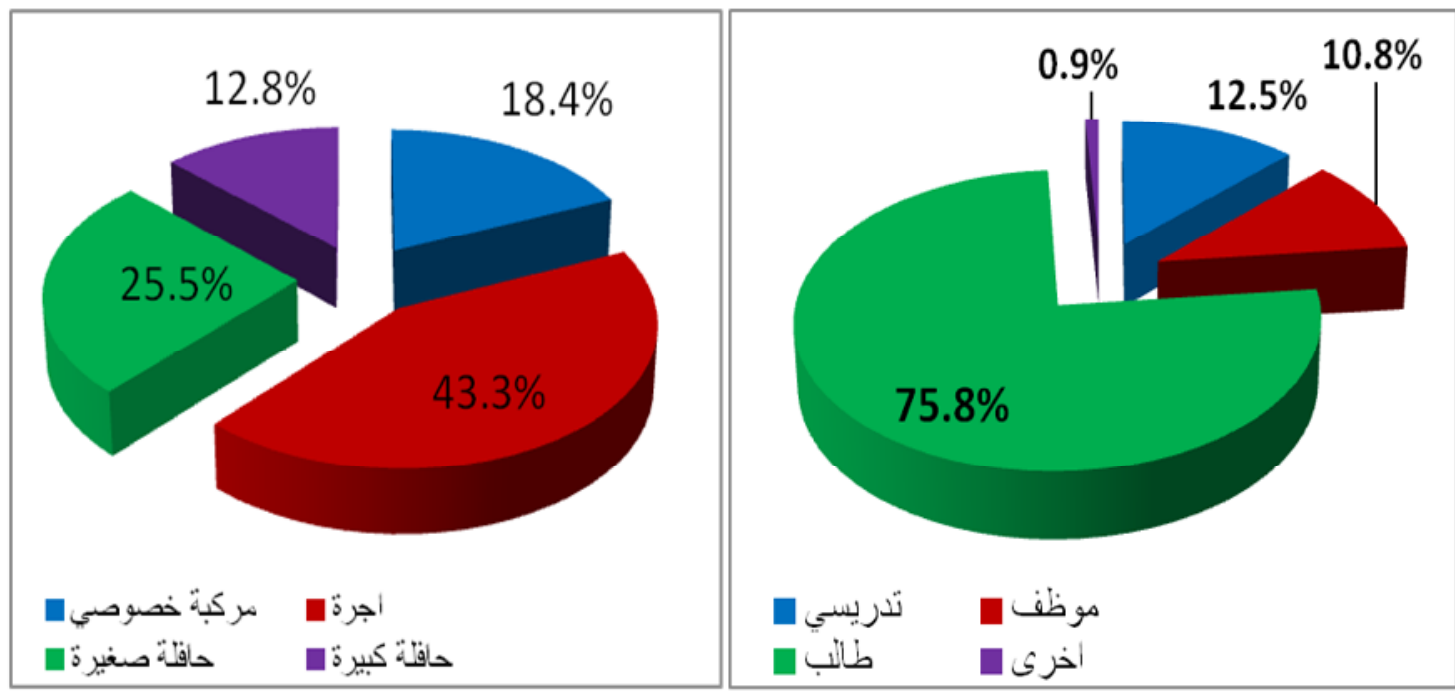

شكل(3): نسب التوزيع و اسطة النقل المستخدمة للوصول شكل (4):نسب التوزيع الفئوي لمستخدمي حافلات

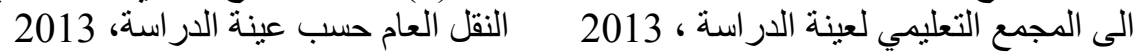

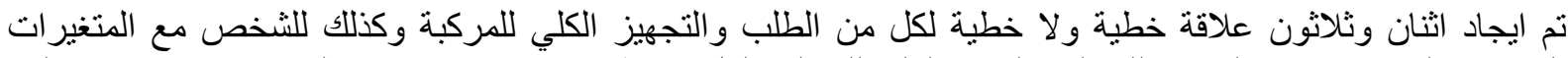

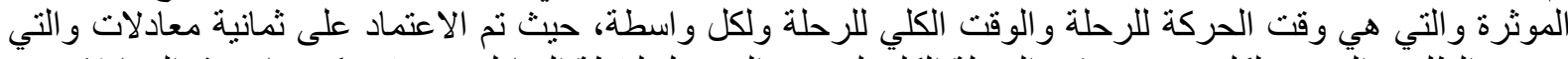

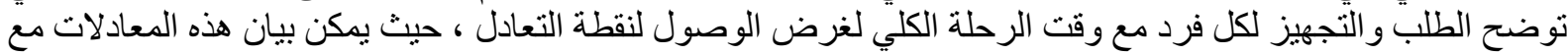

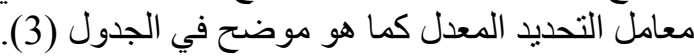

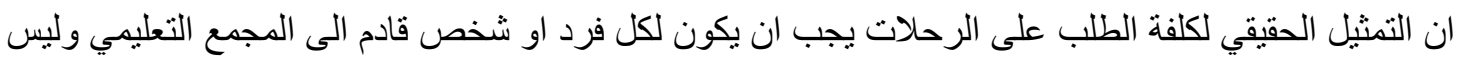

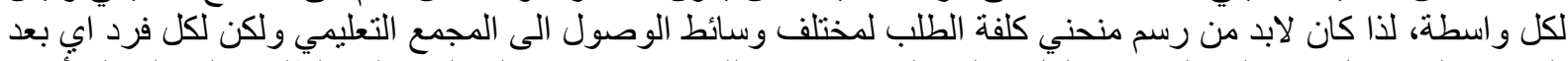

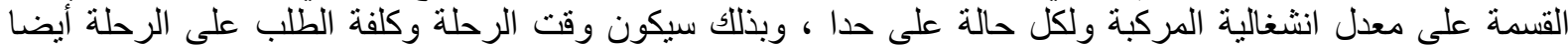

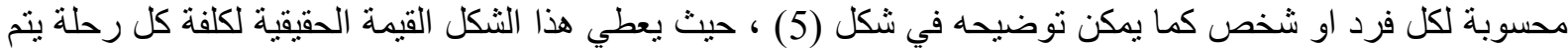
تتفيذها من قبل شخص واحد اي ما يترتب عليه من كلفة حقيقية لكل رحلة دون اعطاء المعادلات.

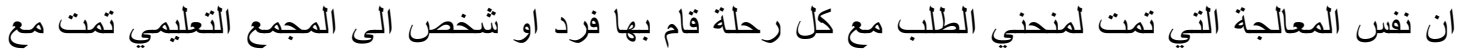

منحنيات التجهيز لكل واسطة مستخدمة، وبذلك ظهرت العلاقة بشكل واضح كما في شكل (6) و الذي بين العلاقة الطردية الخطية لمنفذ الرحلة.

جدول (3) : المعادلات النهائية لعلاقات المتغير المؤثر مع المتغير ات المعتمدة ولكل فرد حسب الو اسطة المستخدمة

\begin{tabular}{|c|c|c|c|c|c|c|}
\hline F - test & $\begin{array}{l}\text { Std. } \\
\text { Error }\end{array}$ & $\begin{array}{c}R^{2} \\
\text { (adj.) }\end{array}$ & Model & $\begin{array}{c}\text { Dependent } \\
\text { Variable }\end{array}$ & $\begin{array}{l}\text { Veh. } \\
\text { Type }\end{array}$ & $\begin{array}{l}\text { Effective } \\
\text { Variable }\end{array}$ \\
\hline $1.961 \mathrm{E} 3$ & 0.650 & 0.779 & $\mathrm{Y} 1 \mathrm{P}=31.928 * 1 / \mathrm{Y} 40$ & الطلب للفرد & خصوصبة & \multirow{8}{*}{$\begin{array}{c}\text { وقت الرحلة } \\
\text { Travel } \\
\text { Time } \\
\text { ( Y40) }\end{array}$} \\
\hline $1.631 \mathrm{E} 3$ & 0.861 & 0.653 & $\mathrm{Y} 1 \mathrm{P}=40.791 * 1 / \mathrm{Y} 40$ & الطلب للفرد & اجرة & \\
\hline 803.9 & 0.272 & 0.616 & $\mathrm{Y} 1 \mathrm{P}=14.522 * 1 / \mathrm{Y} 40$ & الطلب للفرد & حافلة صغيرة & \\
\hline $1.148 \mathrm{E} 3$ & 0.043 & 0.821 & $\mathrm{Y} 1 \mathrm{P}=4.221 * 1 / \mathrm{Y} 40$ & الطلب للفرد & حافلة كبيرة & \\
\hline $2.112 \mathrm{E} 3$ & 0.573 & 0.792 & $\mathrm{Y} 2 \mathrm{P}=0.028 * \mathrm{Y} 40$ & التجهيز للفرد & خصوصية مركبة & \\
\hline $2.161 \mathrm{E} 3$ & 0.312 & 0.713 & $\mathrm{Y} 2 \mathrm{P}=0.011^{*} \mathrm{Y} 40$ & التجهيز للفرد & اجرة & \\
\hline $1.055 \mathrm{E} 3$ & 0.128 & 0.678 & $\mathrm{Y} 2 \mathrm{P}=0.003 * \mathrm{Y} 40$ & التجهيز للفرد & حافلة صغيرة & \\
\hline $3.091 \mathrm{E} 3$ & 0.019 & 0.925 & $\mathrm{Y} 2 \mathrm{P}=0.001 * \mathrm{Y} 40$ & التجهيز للفرد & حافلة كبيرة & \\
\hline
\end{tabular}




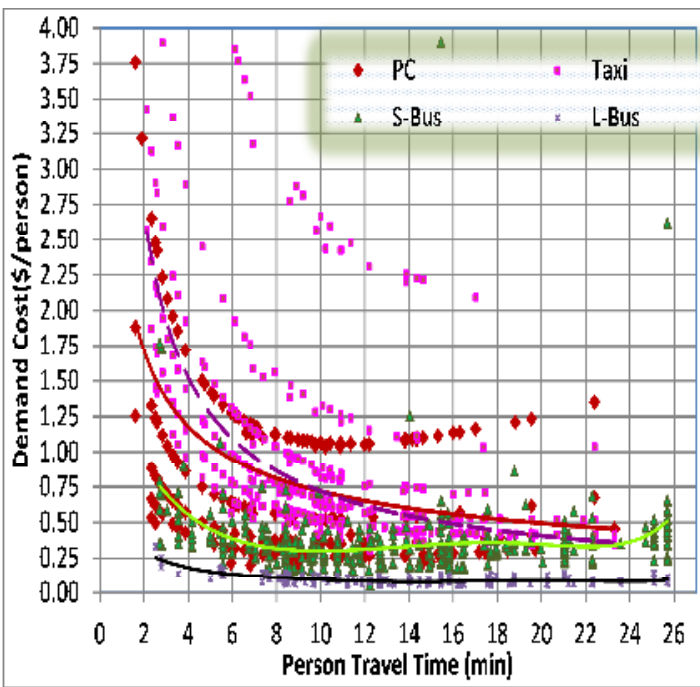

شكل(6) : كلفة التجهيز لكل شخص مع وقت الرحلة

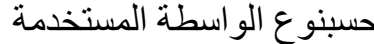

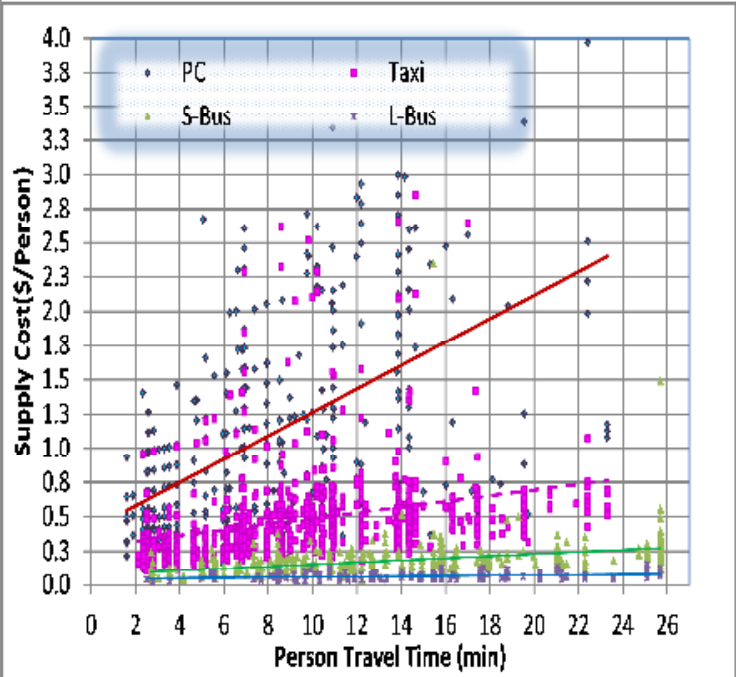

شكل(5) : كلفة الطلب لكل شخص مع وقت الرحلة حسبنوع الو اسطة المستخدمة

ان النتيجة الطبيعية لتمثيل كلفة الرحلات هي ان تتم رسم العلاقات في ان واحد مع جميع المتغيرات المطلوبة وذللك بغية

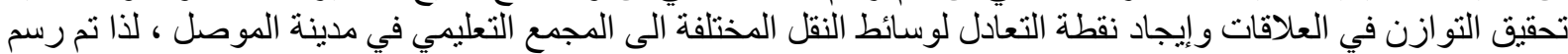

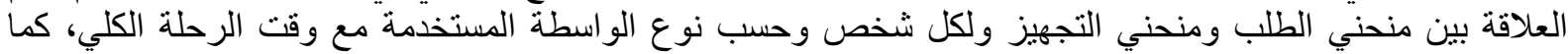
موضح في شكل (7)كما اعطيت معادلاته في جدول (3) سابقأ.

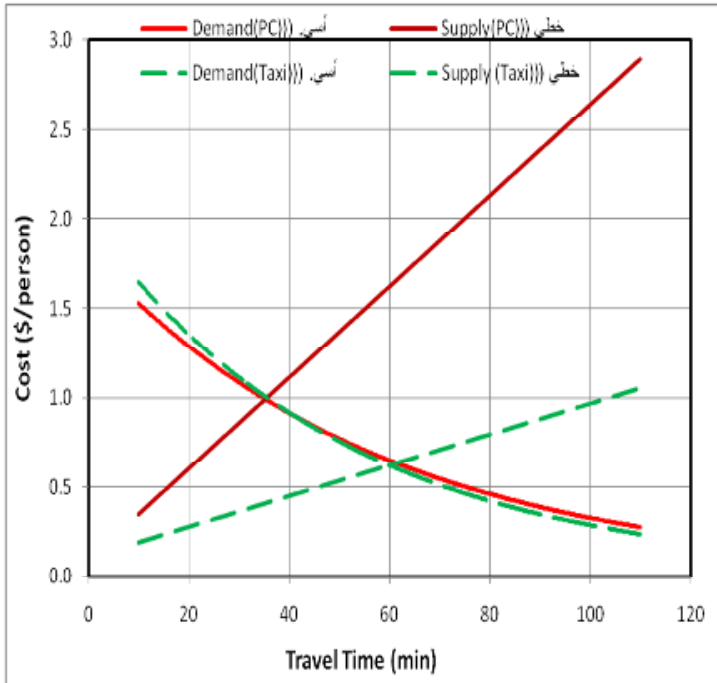

بـ الحافلات الصغيرة و الكبيرة

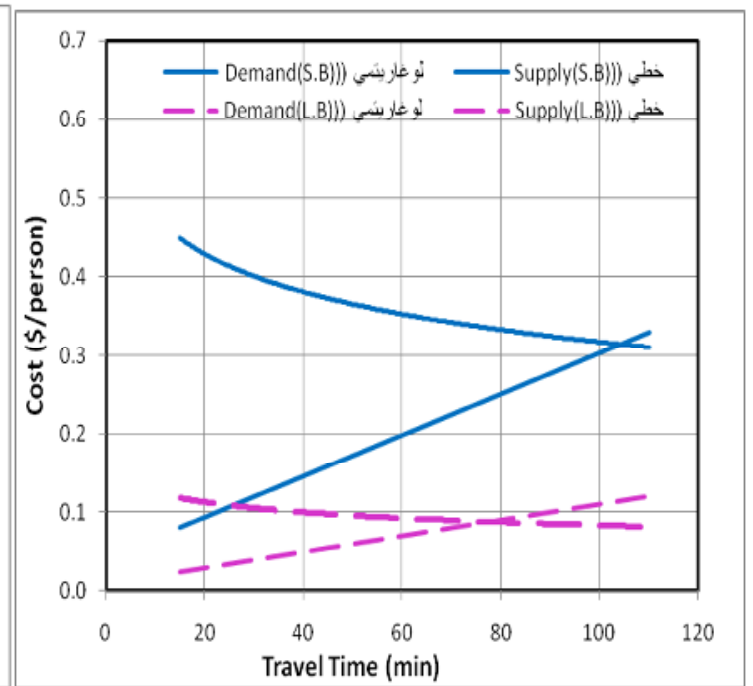

أـ المركبة الخصوصي و الأجرة

شكل (7) : نقطة التعادل حسب نوع الو اسطة التي تبين كلفة النقل لكل فرد مع وقت الرحلة

$$
\text { الاستتتاجات و التوصيات: }
$$

لغرض ايجاد التوازن بين مقدار الطلب على الرحلات والكلف الناتجة عنه وبين مقدار مايتم توفيره من تسهيلات

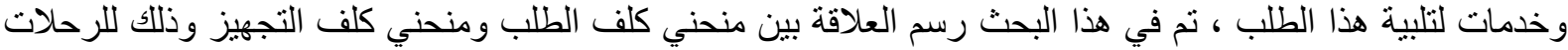

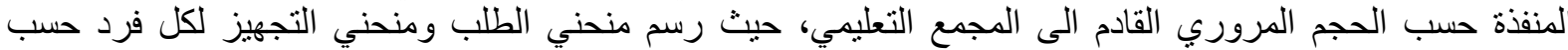

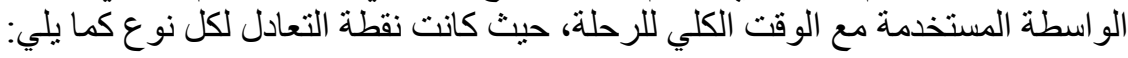




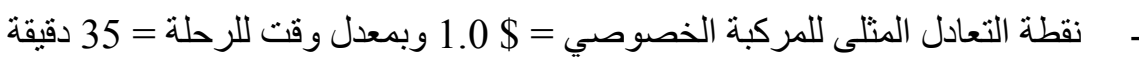

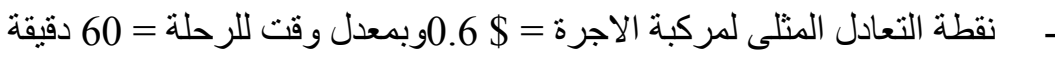

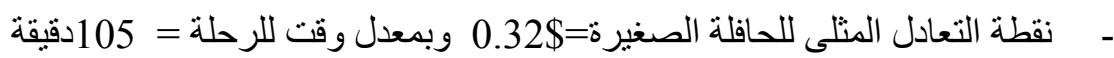

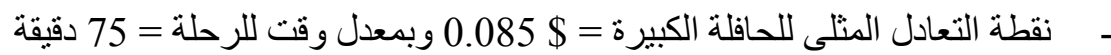

كانت نتائج نقطة التعادل للحافلات بوقت رحلة طويل وهذا منوقع لكون اغلب الحافلات تكون مسافة الرحلة لها كبيرة

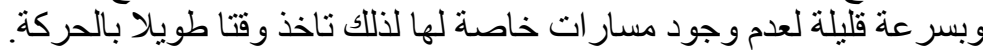

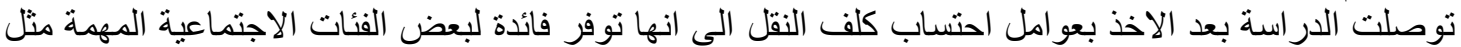

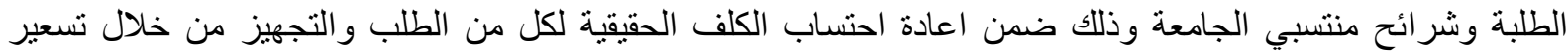

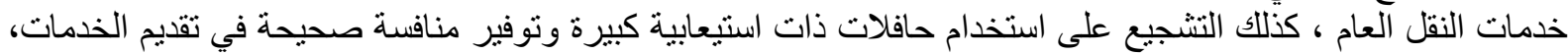
وبذللك يمكن اعطاء التوصيات التالية:

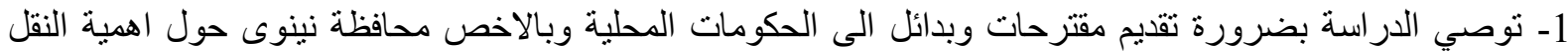

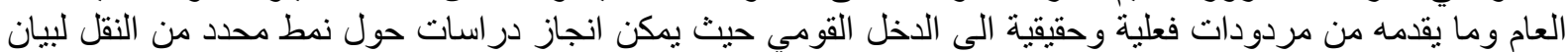

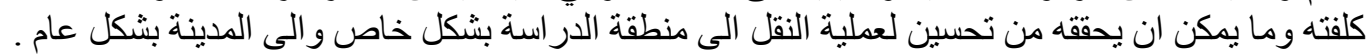

2- توصي الدراسة بضرورة انشاء خطوط جديدة للنقل العام من والى المجمع التعليمي في المدينة وذللك كاحد الحلول

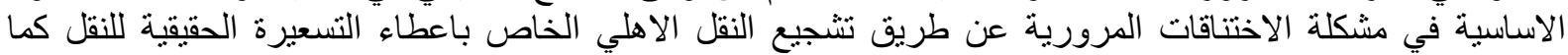

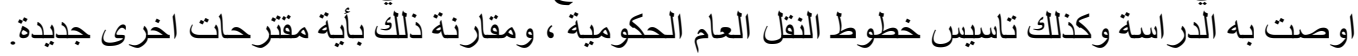

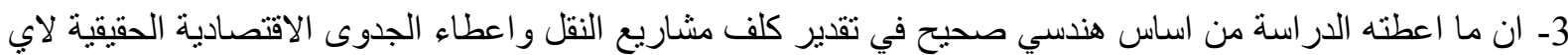

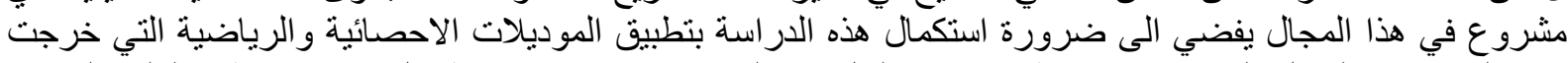

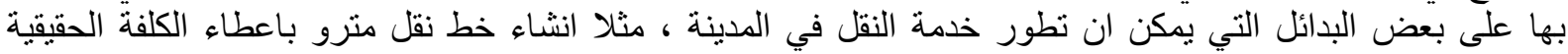

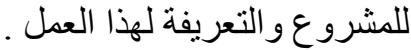

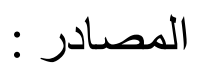

1- Mahmood, Abdul-AlbasetAbdul-Aziz, "Transit Planning Study in the North-Eastern Corridor of Mosul City ", University of Mosul , Collage of Engineering , Department of Civil Engineering, Mosul, Iraq, January, 1984.

2- AL-Kattan, Saleh Abdul-Kareem, " Bus Transit Study for Mosul Higher Education Center", University of Mosul, Collage of Engineering , Department of Civil Engineering , Mosul, Iraq, July, 1986.

3- Naser, Ali F.," Performance Evaluation of Some Public Transport Bus Routes in Baghdad City", University of Technology , Building and Construction Engineering Department, Baghdad, Iraq, March, 2004.

4- ITMP, Iraqi Transport Master Plan, A joint project between Iraqi Government and Italian Government ,C.I.I.T.I. Italian Consortium for Iraqi Transport Infrastructure,( AnasEnac- Enav - FS - Italferr), Draft issue Des.2004, Final - July , 2005.

5- Wright, Paul H. and Ashford, Norman J. "TRANSPORTATION ENGINEERING: PLANNING AND DESIGN ", John Wiley \& Sons. 4th edition. (1998).

6- Salter,R. J., " Highway Traffic Analysis and Design", Reader in civil engineering, University of Bradford, Second Edition, Macmillan, 0-333-48339-1, 1989.

7- Jeff Watson, " How to Determine a Sample Size", Tipsheet \#60, University Park, PA: Penn State Cooperative Extension, http://www.extension.psu.edu/evaluation/pdf/TS60.pdf, 2001. 